\title{
Madurez tecnológica de la generación Z: reto de la transformación digital en Colombia *
}

\author{
Technological Maturity of Generation Z: The Challenge of Digital \\ Transformation in Colombia
}

\author{
Oscar Armando Mejía-Delgado \\ Doctorando en Administración, Universidad del Rosario, \\ Bogotá - Colombia, oscar.mejiad@urosario.edu.co \\ Yury Yasmin Mejía-Delgado \\ Doctorando en Administración, Universidad del Rosario, \\ Bogotá - Colombia, yuri.mejia@urosario.edu.co
}

\begin{abstract}
Cómo citar / How to cite
Mejía-Delgado, O. A., Mejía-Delgado, Y. Y. (2022). Madurez tecnológica de la generación Z: reto de la transformación digital en Colombia. Revista CEA, v. 8, n. 16, e1913.

https://doi.org/10.22430/24223182.1913
\end{abstract}

Recibido: 17 de mayo de 2021

Aceptado: 13 de septiembre de 2021

\section{Resumen}

Las diferencias generacionales son consideradas la causa principal que determina la capacidad de interacción con nuevas tecnologías. El concepto de «nativo digital» ha tomado fuerza y visibilidad en ciertas áreas de conocimiento tales como ingenierías y tecnología, ciencias de la salud, ciencias sociales y administrativas, entre otras. Por tal motivo, el objetivo principal de esta investigación fue determinar la madurez digital de la generación Z en Colombia, a partir de la correlación existente entre el conocimiento sobre la transformación digital, la apropiación de tecnologías digitales, la experiencia e intención de uso de herramientas digitales en el futuro. El enfoque del estudio fue cuantitativo con un diseño no experimental, de carácter transversal, donde además se realizó un cuestionario como instrumento de recolección de datos, el cual fue suministrado, vía internet, a 250 personas. Entre los principales hallazgos se encontró que las variables de análisis como el conocimiento sobre la transformación digital, la experiencia de uso de herramientas digitales y la intención de uso en el futuro, aportan y están relacionadas de forma significativa con la madurez tecnológica de la generación Z. La evidencia proporcionada valida el comportamiento tradicional de adopción de nuevas tecnologías, donde, esta investigación contribuye al área de administración y

\footnotetext{
* Este artículo se deriva de la tesis doctoral «Madurez tecnológica de la generación Z: reto de la transformación digital en Colombia» y ha sido financiado con recursos propios.
} 
gestión tecnológica argumentando que el nivel de madurez tecnológica de la generación Z impacta de manera directa cualquier estrategia de transformación digital empresarial.

Palabras clave: madurez digital, nativos digitales, transformación digital, generación Z.

Clasificación JEL: 014, O33.

\title{
Highlights
}

- La adopción de nuevas tecnologías digitales por parte de la generación Z puede generar mayor competitividad empresarial.

- La madurez tecnológica de la generación Z puede influenciar el éxito en la implementación de herramientas digitales en las organizaciones.

- Incrementar el conocimiento de nuevas tecnologías digitales en la generación Z puede generar mayor perdurabilidad y sostenibilidad empresarial.

\begin{abstract}
Generational differences are considered the main cause that determines the ability to interact with new technologies. The concept of digital native has gained strength and visibility in certain fields of knowledge such as engineering and technology, health sciences, and social and management sciences, among others. The main objective of this study was to determine the digital maturity of Generation $Z$ in Colombia based on the existing correlation between knowledge on digital transformation, the appropriation of digital technologies, and the experience and intention to use digital tools in the future. The research approach of this study was quantitative with a nonexperimental, cross-sectional design, where a survey was administered via the Internet to 250 people as a data collection instrument. Among the main findings, it was determined that the variables under analysis - such as knowledge on digital transformation, the experience of using digital tools, and the intention to use them in the future-contribute and are significantly related to the technological maturity of Generation Z. The evidence in this paper validates the traditional behavior of new technology adoption and contributes to the area of management and technology management because it argues that the level of technological maturity of Generation Z directly impacts any digital transformation strategy.
\end{abstract}

Keywords: Digital maturity, digital natives, digital transformation, Generation Z.

JEL classification: $\mathrm{O} 14, \mathrm{O} 33$.

\section{Highlights}

- Generation Z's adoption of new digital technologies can generate greater business competitiveness.

- The technological maturity of Generation Z can influence the success in the implementation of digital tools by organizations.

- Increasing the knowledge of new digital technologies in Generation Z can generate greater business durability and sustainability. 


\section{INTRODUCCIÓN}

A pesar de que se cree que las nuevas generaciones poseen y desarrollan habilidades tecnológicas innatas, algunos autores denominan a los nacidos después de 1980 como nativos digitales, ya que surgieron rodeados de nuevas tecnologías (Verčič y Verčič, 2013; Gurung y Rutledge, 2014), lo que los hace más sofisticados con respecto a generaciones anteriores en el uso de internet y dispositivos móviles (Bond et al., 2018). De igual forma son multitarea, es decir que necesitan desarrollar diferentes asignaciones al mismo tiempo: los nativos digitales tienden a aburrirse rápidamente si su trabajo es sistemático (Akçayir et al., 2016), entonces, si la edad es el factor determinante para realizar una clasificación de la adopción de nuevas tecnologías, la brecha entre los nativos digitales y las demás generaciones migrantes nunca podrá cerrarse (Helsper y Eynon, 2010).

Para Helsper y Eynon, existen unos estereotipos que clasifican a los jóvenes que estudian en las escuelas, colegios y universidades: primero, nativos digitales; segundo, generación de la red; tercero, generación Google; términos acuñados para resaltar la importancia de las nuevas tecnologías en la vida a través de la cual hoy los jóvenes se comunican, socializan, crean y aprenden (Helsper y Eynon, 2010). El gran desarrollo de tecnologías de la información ha inducido a grandes cambios en los procesos de las organizaciones y más específicamente en países en vía de desarrollo (Hadullo et al., 2017).

Es así como la mayoría de los líderes empresariales reconocen que la transformación digital (TD) es un tema crucial y crítico, pero tan solo la mitad de ellos tiene el conocimiento de la TD y su proceso de implementación; por esta razón, las empresas se encuentran en diferentes niveles de maduración digital (Mugge et al., 2020), por lo que se requieren profesionales con conocimientos y apropiaciones digitales capaces de afrontar los retos que presenta la TD (Kuzin, 2019). Ahora bien, las interacciones entre gobierno, empresa y academia son fundamentales para crear las condiciones necesarias e innovar en una sociedad basada en el conocimiento (Pappas et al., 2018; Matt, 2020), en tanto la TD permite, a través de sus tecnologías digitales, generar valor a las sociedades y mejorar sus condiciones de vida.

En consecuencia, todas las organizaciones que quieran participar y competir en la economía digital tendrán que realizar inversiones en transformación de procesos, talento humano, tecnología de recolección y análisis de datos (Pappas et al., 2018; Kumar et al., 2021), lo que permitirá crear una ventaja competitiva a partir de la capacidad de análisis de grandes datos (Big data) (Kisimov et al., 2020; De Luca et al., 2021), donde una empresa podrá capturar, almacenar y analizar datos, lo que le permitirá crear valor y un cambio estratégico en las organizaciones y en la sociedad (Pappas et al., 2018).

Además, se deben alinear los procesos de enseñanza-aprendizaje con las necesidades futuras de las organizaciones; por ejemplo, las universidades deben diseñar metodologías donde las personas se sientan apoyadas en el desarrollo de sus competencias y preparados para los futuros desafíos laborales (LeBlanc, 2018). Por lo anterior, este tema atrae cada vez más la atención de empresarios, expertos e investigadores, especialmente en términos no solo de implementación de tecnología, sino preferiblemente en competencias, habilidades y gestión tecnológica. 
En este orden de ideas, el propósito de este estudio es determinar el nivel de madurez digital de la generación Z, a partir de la relación que existe entre el conocimiento de la transformación digital, la apropiación de herramientas digitales, la experiencia de uso de tecnologías digitales y la intención de uso en el futuro de herramientas digitales, lo cual permitirá a instituciones de educación superior (IES) y organizaciones de diferentes sectores económicos comprender el nivel de maduración digital que poseen los futuros responsables de los procesos de la TD.

Este artículo se divide en cuatro grandes secciones: la primera es la introducción, en la cual se podrá consultar la revisión de literatura e identificar las variables de análisis que permiten construir el marco de referencia para el planteamiento de hipótesis; en la segunda, se encuentra la metodología, donde, se plantean las hipótesis y el modelo de investigación conceptual, asimismo, se describe el instrumento de recolección de datos y la operacionalización de las variables; en la tercera sección se presenta el análisis de resultados, donde se da respuesta a las hipótesis formuladas; la cuarta y última sección, presenta el capítulo de discusión y las principales conclusiones, donde se indican las limitaciones del estudio y se plantean futuras líneas de investigación.

\section{MARCO TEÓRICO}

\section{Aproximación a la transformación digital}

En principio llegar a una definición compartida de TD no resulta fácil. Algunos investigadores abordan el concepto desde el cambio organizacional o estratégico y se apoyan en algunas tecnologías como el big data para argumentar su postulado, mientras, algunos autores abordan la TD, no solamente como el cambio tecnológico, sino también miden el impacto que tiene al interior de las organizaciones (Hausberg et al., 2019). Lo anterior conduce a que haya una transformación en todas las actividades claves de la empresa y afecta de una manera directa las estructuras organizaciones y las formas de concebir la gestión empresarial (Matt et al., 2015).

Por otra parte, la rápida evolución que han tenido las tecnologías de la información, comunicación y conectividad en la última década, ha permitido que las empresas aprovechen y den respuesta a entornos turbulentos (Bharadwaj et al., 2013). Las tecnologías digitales están transformando la estructura de las relaciones humanas a través de las redes sociales (Zhou y Xu, 2007), lo que permite desarrollar nuevos modelos de negocio y estrategias globales. La estrategia de TD ya no solo apoya o refuerza la estrategia comercial, sino que además es un eslabón fundamental en la estrategia corporativa, ya que las tecnologías de la información (TI) son utilizadas en todas las áreas funcionales de la organización, tales como, operaciones, abastecimiento, producción, distribución, marketing y comercialización (Conner y Prahalad, 1996).

En consecuencia, la TD se refiere a la combinación de varias innovaciones tecnológicas y que trae consigo nuevos actores, estructuras, practicas, valores y creencias que cambian y transforman practicas actuales dentro de las organizaciones (Hinings et al., 2018), por lo tanto, la TD es la adaptación de las empresas a una nueva realidad creada por las nuevas tecnologías, las cuales han arrasado con empresas que no estaban preparadas para este cambio, pues están transformando todo lo que se conocía o se creía era eficiente (Kane, 2019). 
Sin embargo, la TD necesita desarrollar su propia estrategia, que servirá como concepto central para integrar todas las operaciones de la organización (Seong Leem et al., 2008). Por lo general, la integración de tecnologías digitales afecta a todos los eslabones de una cadena de suministro, pero igualmente trae beneficios a mediano y largo plazo, como el aumento en los ingresos, incremento en la productividad, innovaciones en la creación de valor, formas de interactuar con los clientes, entre otros (Matt et al., 2015), además se concibe la TD como el uso de la tecnología para mejorar radicalmente el rendimiento, productividad y resultados de la organización, donde se identifican tres eslabones en los que se concentra el esfuerzo de una TD de una empresa: experiencia del cliente, procesos operacionales y modelo de negocio (El Hilali et al., 2020).

La TD impulsa los cambios que provocan las tecnologías digitales en los modelos de negocio que da como resultado la automatización de procesos (Stjepić et al., 2020). Estos cambios se pueden observar en la demanda que se genera a través de internet y en los procesos de comunicación que emplean las organizaciones (Matt et al., 2016).

\section{Nuevas tecnologías digitales}

Desarrollar y gestionar una ventaja competitiva es un reto para las organizaciones y más hoy, cuando las tecnologías digitales son para muchas empresas un tema poco conocido y explotado, los mejores resultados los obtienen empresas que poseen modelos globales y estructuras tecnológicas sólidas (Adamik y Nowicki, 2018). El concepto de industria 4.0 es acertado para explicar la revolución tecnológica de los últimos años, el cual se basa en una serie de tecnologías (ver Figura 1).

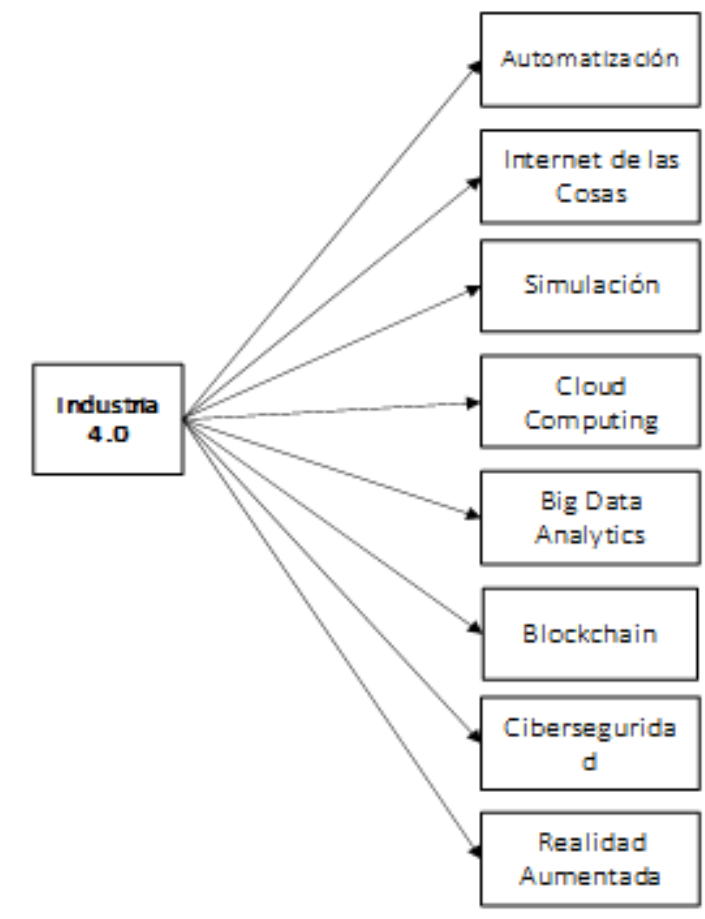

Figura 1. Tecnologías de la Industria 4.0

Figure 1. Industry 4.0 technologies

Fuente: elaboración propia. 
Para desarrollar un proceso de TD exitoso se necesita que los individuos creen y perciban la utilidad de la tecnología, comprender que la TD mejora su rendimiento. La creencia de que la tecnología es fácil de usar permitirá implementar las tecnologías digitales de una forma más sencilla y orgánica dentro de la organización (Roumani et al., 2015). La industria 4.0 plantea cuatro grandes desafíos para la TD: primero, las grandes inversiones financieras que una organización debe estar dispuesta a realizar en automatización y TIC; segundo, la transformación del modelo de negocio, el cual necesitará de mayor colaboración entre diferentes actores o posibles nuevos socios, por ejemplo, proveedores de servicios de telecomunicaciones e internet; tercero, la recolección y análisis de grandes cantidades de datos necesitan confidencialidad y una política clara de protección de datos; y cuarto, el desafío que gira alrededor de los problemas legales, responsabilidades de producto y propiedad intelectual que debe abordarse (Fonseca, 2018).

\section{Conocimiento sobre transformación digital}

Se debe preparar a los ciudadanos con las competencias necesarias en el uso de las nuevas tecnologías digitales de manera crítica y creativa (Blayone et al., 2018; Redecker, 2017), dado que el desarrollo de estas habilidades hace que la compensación salarial sea mayor a los que no las poseen (Hajkowicz et al., 2016).

\section{Apropiación tecnologías digitales}

Por otra parte, la TD produce grandes cambios sistémicos en la sociedad, en la estructura de las instituciones y en el comportamiento e interacción de los objetos con el entorno; asimismo, la transformación digital se entiende como la transformación de todo un modelo de negocio, dicho de otra forma, afecta la estrategia, procesos y la estructura organizacional, (Ipatov et al., 2020).

Igualmente, las tecnologías digitales traen consigo beneficios para las empresas que toman la decisión de realizar una transformación digital, por ejemplo, desarrollar nuevos productos, servicios, procesos e incluso un cambio en el modelo de negocio (Hinings et al., 2018; Newman y Beetham, 2017).

\section{Experiencias de uso de tecnologías digitales}

Entonces, para poder determinar el nivel de maduración tecnológica que posee una organización se debe determinar las posibles barreras que pueden existir al momento de implementar una estrategia de TD, donde todas las partes interesadas participan activamente y se involucran en el proceso asumiendo responsabilidades y desafíos (Ifenthaler y Egloffstein, 2020). La principal barrera habitualmente se encuentra en compartir una visión común por parte del equipo responsable, es necesario tener claridad y comprensión de los objetivos (Machika y Dolley, 2018).

La transformación digital ha generado cambios en los procesos de aprendizaje de los individuos y de las organizaciones; conceptos como: (i) internet, (ii) disrupción, (iii) nativos digitales, (iv) competencias digitales, (v) aprendizaje profundo, (vi) industria 4.0 generarán grandes cambios futuros (Kamsker et al., 2020). 


\section{METOdOLOGÍA}

\section{Formulación de hipótesis}

El modelo de investigación se basa en determinar el nivel de maduración digital de la generación Z, aunque son varios los estudios que se concentran en modelos de madurez tecnológica organizacional (Ifenthaler y Egloffstein, 2020; Seong Leem y Kim, 2004; Putri y Muljoredjo, 2014), son escasos los que se centran en comprender al usuario y gestor de nuevas tecnologías, desde el conocimiento que tiene de la transformación digital hasta la experiencia e intención de uso de herramientas digitales (Roumani et al., 2015).

$H_{1}$ : Existe una relación positiva entre el conocimiento sobre transformación digital y apropiación de las nuevas tecnologías digitales.

$H_{2}$ : Existe una relación positiva entre el conocimiento sobre transformación digital y la experiencia de uso de herramientas digitales.

$H_{3}$ : Existe una relación positiva entre el conocimiento sobre transformación digital y la intención de uso en el futuro de herramientas digitales.

por lo tanto, se plantean las siguientes hipótesis:

$H_{4}$ : Existe una relación positiva entre la apropiación de las nuevas tecnologías digitales y la experiencia de uso de herramientas digitales.

$H_{5}$ : Existe una relación positiva entre la apropiación de las nuevas tecnologías digitales y la intención de uso en el futuro de herramientas digitales.

$H_{6}$ : Existe una relación positiva entre la experiencia en el uso de herramientas digitales y la intención de uso en el futuro.

\section{Instrumento y recolección de información}

Este estudio fue de carácter cuantitativo, no experimental, la muestra estaba conformada por 129 mujeres (51.6\%) y 121 hombres (48.4\%), para un total de 250 encuestados, con una edad promedio de 20.15 años ( $D E=1.65)$, se consideraron personas ubicadas en la ciudad de Bogotá, los encuestados pertenecen al estrato medio-alto de la escala social de Colombia, la muestra corresponde a la generación Z, los cuales tienen una mayor inclinación por las nuevas tecnologías digitales, los motivos de la investigación y el modelo de investigación no se compartió con los encuestados antes de responder el cuestionario, esto con el ánimo de no sesgar sus respuestas, los datos fueron recolectados entre octubre y noviembre de 2020.

El cuestionario contenía dieciséis afirmaciones, incluyendo cuatro variables de control, tres preguntas sobre datos demográficos, cada pregunta fue medida a través de una escala Likert de cinco puntos: (1) totalmente en desacuerdo a (5) totalmente de acuerdo. El cuestionario fue adaptado al contexto y modificado de la revisión de literatura; en cuanto a la variable conocimiento sobre la transformación digital, cuyos principales referentes son Blayone et al. 2018; Gefen, 2000, sus estudios se basan en el conocimiento que posee el usuario final en las plataformas de comercio electrónico, las variables apropiación de tecnologías digitales e intenciones de uso en el futuro, que tomaron como referente a Hinings et al., 2018; Ifenthaler y Egloffstein, 2020,sus investigaciones se basan en desarrollar un modelo holístico de madurez tecnológica, que identificó los factores que median la madurez digital y el desempeño organizacional (motivos y actitudes); por otra parte, determinó la importancia de la 
cultura organizacional para la adopción de nuevas tecnologías digitales; finalmente, la variable experiencia de uso de herramientas digitales que toma como referente a Ifenthaler y Egloffstein, 2020, en sus estudios se refiere a la necesidad de experimentar previamente que las nuevas tecnologías para afianzar su uso en el futuro, (ver Tabla 1).

Tabla 1. Operacionalización de variables

Table 1. Operationalization of variables

\begin{tabular}{|c|c|c|c|}
\hline Variable & Clave & Definición & Referencias clave \\
\hline $\begin{array}{l}\text { Conocimiento } \\
\text { transformación } \\
\text { digital }\end{array}$ & CONTD & $\begin{array}{c}\text { Conocimientos y habilidades que apoyan el } \\
\text { uso efectivo de la tecnología }\end{array}$ & $\begin{array}{l}\text { Literatura sobre } \\
\text { transformación digital } \\
\text { (Blayone et al., 2018) }\end{array}$ \\
\hline $\begin{array}{l}\text { Apropiación } \\
\text { tecnologías } \\
\text { digitales }\end{array}$ & APDI & $\begin{array}{c}\text { Entendimiento de las herramientas que } \\
\text { permiten desarrollar nuevos productos, } \\
\text { procesos, servicios e incluso nuevos modelos } \\
\text { de negocio }\end{array}$ & $\begin{array}{l}\text { Literatura sobre innovación } \\
\text { digital (Hinings et al., 2018, } \\
\text { 2018) }\end{array}$ \\
\hline $\begin{array}{l}\text { Experiencia de } \\
\text { uso } \\
\text { herramientas } \\
\text { digitales }\end{array}$ & EXTD & $\begin{array}{l}\text { Experiencia sobre el uso efectivo de } \\
\text { tecnologías digitales }\end{array}$ & $\begin{array}{l}\text { Literatura sobre modelos de } \\
\text { madurez tecnologica } \\
\text { (Ifenthaler y Egloffstein, } \\
\text { 2020) }\end{array}$ \\
\hline $\begin{array}{l}\text { Intenciones de } \\
\text { uso en el } \\
\text { futuro }\end{array}$ & INTUF & $\begin{array}{c}\text { Voluntad de usar en un futuro las } \\
\text { tecnologías digitales para su desarrollo } \\
\text { profesional y laboral }\end{array}$ & $\begin{array}{c}\text { Literatura sobre innovación } \\
\text { digital (Hinings et al., 2018, } \\
\text { 2018) }\end{array}$ \\
\hline
\end{tabular}

A partir de lo anterior, se solicitó la participación voluntaria de los encuestados y se procedió a suministrar el cuestionario vía internet a 250 personas, donde inicialmente se les solicitó su consentimiento para el uso de los datos. La tasa de respuesta fue del $100 \%$.

\section{RESULTADOS}

Para realizar los análisis estadísticos de los resultados se utilizó el programa IBM SPSS Statistics, versión 26. Inicialmente se realizó el análisis de fiabilidad del instrumento, el valor del Alfa de Cronbach fue 0.875 , lo que significa una muy buena consistencia del instrumento, las principales características de la muestra se presentan (ver Tabla 2).

Tabla 2. Características principales de los encuestados Table 2. Main features of the respondents

\begin{tabular}{ccccc}
\hline & & Frecuencia & Porcentaje válido & $\begin{array}{c}\text { Porcentaje } \\
\text { acumulado }\end{array}$ \\
\hline \multirow{2}{*}{ Género } & Masculino & 121 & $48.4 \%$ & $48 \%$ \\
\cline { 2 - 5 } & Femenino & 129 & $51.6 \%$ & $100 \%$ \\
\hline Edad & Total & 250 & $100 \%$ & $100 \%$ \\
\hline
\end{tabular}

Fuente: elaboración propia. 


\section{Análisis factorial exploratorio}

Para poder realizar un análisis factorial exploratorio (AFE), se llevó a cabo la prueba de KMO y Bartlett, esta prueba se realiza con el objetivo de comprobar si es posible realizar un AFE o no. Un valor aceptable para esta prueba es superior a 0.60 , el resultado para este grupo de datos fue 0.846 , lo cual se considera muy bueno, la prueba de Bartlett es altamente significativa $p=0.000$, lo que quiere decir que existe una alta correlación entre los factores, por lo tanto, realizar un AFE es adecuado (ver Tabla 3).

Tabla 3. Prueba de KMO y Bartlett

Table 3. KMO and Bartlett's test

\begin{tabular}{|c|c|c|}
\hline \multicolumn{2}{|c|}{$\begin{array}{l}\text { Medida Kaiser-Meyer-Olkin de adecuación de } \\
\text { muestreo }\end{array}$} & \multirow{2}{*}{$\begin{array}{c}0.846 \\
2134.50\end{array}$} \\
\hline Prueba de esfericidad de Bartlett & $\begin{array}{l}\text { Aprox. Chi- } \\
\text { cuadrado }\end{array}$ & \\
\hline & gl & 120 \\
\hline & Sig. & 0.000 \\
\hline
\end{tabular}

Fuente: elaboración propia.

A continuación, con el objetivo de realizar un análisis de correlaciones entre factores, el AFE agrupó el conjunto de datos en cuatro variables, las cuales explican el $69.84 \%$ de la varianza (Churchill, 1979), (ver Tabla 4).

Tabla 4. Varianza total explicada

Table 4. Total variance explained

\begin{tabular}{cccccccccc}
\hline Ítem & Total & $\begin{array}{c}\% \text { de } \\
\text { varianza }\end{array}$ & $\begin{array}{c}\% \\
\text { acumulado }\end{array}$ & Total & $\begin{array}{c}\% \text { de } \\
\text { varianza }\end{array}$ & $\begin{array}{c}\% \\
\text { acumulado }\end{array}$ & Total & $\begin{array}{c}\% \text { de } \\
\text { varianza }\end{array}$ & $\begin{array}{c}\% \\
\text { acumulado }\end{array}$ \\
\hline 1 & 4.985 & 31.15 & 31.154 & 4.985 & 31.154 & 31.154 & 3.355 & 20.971 & 20.971 \\
\hline 2 & 3.646 & 22.78 & 53.940 & 3.646 & 22.786 & 53.940 & 3.109 & 19.433 & 40.403 \\
\hline 3 & 1.477 & 9.233 & 63.173 & 1.477 & 9.233 & 63.173 & 2.877 & 17.980 & 58.384 \\
\hline 4 & 1.068 & 6.674 & 69.847 & 1.068 & 6.674 & 69.847 & 1.834 & 11.463 & 69.847 \\
\hline \multicolumn{10}{c}{ Método de extracción: análisis de componentes principales. } \\
\hline \multicolumn{10}{c}{ Fuente: elaboración propia. }
\end{tabular}

Así, se revisó la matriz de correlación de ítems, donde se aplicó un método de extracción de principales factores y una rotación Varimax con normalización Kaiser, esto con el objetivo de garantizar la relación de los ítems que conforman cada variable (Ha y Stoel, 2009), (ver Tabla 5). 
Tabla 5. Resultados del análisis factorial exploratorio (matriz de patrones) Table 5. Results of the exploratory factor analysis (pattern matrix)

\begin{tabular}{|c|c|c|c|c|c|}
\hline & Item & APDI & INTUF & CONTD & EXTD \\
\hline \multirow{4}{*}{ Conocimiento transformación digital } & CONTD01 & & & 0.727 & \\
\hline & CONTD02 & & & 0.801 & \\
\hline & CONTD03 & & & 0.809 & \\
\hline & CONTD04 & & & 0.828 & \\
\hline \multirow{5}{*}{ Apropiación tecnologías digitales } & APDI01 & 0.740 & & & \\
\hline & APDI02 & 0.848 & & & \\
\hline & APDI03 & 0.782 & & & \\
\hline & APDI04 & 0.736 & & & \\
\hline & APDI05 & 0.831 & & & \\
\hline \multirow{4}{*}{$\begin{array}{l}\text { Experiencia de uso herramientas } \\
\text { digitales }\end{array}$} & EXTD01 & & & & 0.651 \\
\hline & EXTD02 & & & & 0.869 \\
\hline & EXTD03 & & & & 0.476 \\
\hline & EXTD04 & & & & 0.556 \\
\hline \multirow{3}{*}{ Intenciones de uso en el futuro } & INTUF01 & & 0.907 & & \\
\hline & INTUF02 & & 0.883 & & \\
\hline & INTUF03 & & 0.897 & & \\
\hline \multicolumn{6}{|c|}{ Método de extracción: análisis de componentes principales. } \\
\hline \multicolumn{6}{|c|}{ Método de rotación: Varimax con normalización Kaiser.a } \\
\hline
\end{tabular}

En las medias y desviaciones estándar de las respuestas, según el género de los encuestados, se puede observar cómo el conocimiento sobre la transformación digital tiene un valor promedio inferior a 4.0 en ambos géneros. Al observar la diferencia entre hombres y mujeres de la variable apropiación de tecnologías digitales, se encuentra que las mujeres tienen un valor promedio inferior a 2.0, mientras los hombres están por encima de este umbral (ver Tabla 6).

Con respecto a la experiencia de uso de herramientas de transformación digital, se encuentra que el valor promedio en ambos géneros está cerca a los 4.0; para la intención de uso en el futuro se encuentra que el valor promedio es superior a 3.0 en ambos géneros.

La distribución de los datos es importante para determinar el método de estimación, para lo cual fue necesario realizar una prueba de normalidad de datos de Kolgomorov-Smirnov, con el objetivo de establecer el tipo de prueba para comprobar las hipótesis planteadas. Los resultados obtenidos en la prueba de normalidad, donde $p<0.05$, demuestran que las variables no se distribuyen normalmente, lo cual indica que la prueba a utilizar será no paramétrica, la decisión fue emplear el coeficiente de correlación de Spearman (Rho). 
Tabla 6. Estadística descriptiva de acuerdo con el género de los encuestados Table 6. Descriptive statistics classified by respondents' gender

\begin{tabular}{ccccc}
\hline Ítem & Media & Desv Stand & Media & Desv Stand \\
\hline CONTD01 & 3.68 & 0.92 & 3.78 & 0.98 \\
\hline CONTD02 & 3.36 & 0.99 & 3.46 & 0.98 \\
\hline CONTD03 & 3.47 & 0.88 & 3.47 & 0.93 \\
\hline CONTD04 & 3.45 & 0.91 & 3.41 & 0.97 \\
\hline APDI01 & 2.40 & 1.27 & 2.27 & 1.41 \\
\hline APDI02 & 2.06 & 1.25 & 1.84 & 1.15 \\
\hline APDI03 & 2.09 & 1.22 & 2.01 & 1.23 \\
\hline APDI04 & 2.02 & 1.24 & 1.88 & 1.11 \\
\hline APDI05 & 1.89 & 1.23 & 1.78 & 1.15 \\
\hline EXPTD01 & 4.10 & 0.85 & 4.47 & 0.72 \\
\hline EXPTD02 & 4.07 & 0.86 & 4.29 & 0.82 \\
\hline EXPTD03 & 3.91 & 0.96 & 3.99 & 0.91 \\
\hline EXPTD04 & 3.66 & 0.97 & 3.96 & 0.94 \\
\hline INTUF01 & 3.81 & 1.12 & 3.97 & 1.09 \\
\hline INTUF02 & 4.12 & 0.93 & 4.28 & 0.85 \\
\hline INTUF03 & 3.81 & 1.08 & 4.22 & 0.90 \\
\hline & & Fuente: elaboración propia. & & \\
\hline
\end{tabular}

\section{Correlación de Spearman (Rho)}

Esta investigación analizó la correlación existente entre el conocimiento sobre transformación digital y la apropiación de nuevas tecnologías digitales, de igual forma, se buscaba determinar si existe una relación positiva entre el conocimiento sobre la TD y la experiencia en el uso de herramientas digitales, de igual forma se buscó determinar la relación entre el conocimiento sobre TD y la intención de uso en el futuro. Otra correlación que se calculó fue entre la apropiación de tecnologías digitales y la experiencia en el uso de herramientas digitales, por lo tanto, era necesario determinar la relación entre apropiación de tecnologías digitales e intención de uso en el futuro. Finalmente fue importante para este estudio determinar la correlación entre experiencia en el uso de herramientas digitales y la intención de uso en el futuro.

La Tabla 7 evidencia el p-valor obtenido (0.000), que valida la presencia de correlación entre las variables de estudio. De igual forma, se observa el coeficiente de Spearman, donde el resultado fue de 0.474 , lo cual significa una correlación positiva moderada entre las variables analizadas. 
Tabla 7. Correlación de Spearman entre el conocimiento transformación y apropiación digitales Table 7. Spearman's correlation between digital transformation knowledge and digital appropriation

\begin{tabular}{|c|c|c|c|c|}
\hline & & & CONTD & APDI \\
\hline \multirow{6}{*}{ Rho de Spearman } & \multirow{3}{*}{ CONTD } & $\begin{array}{c}\text { Coeficiente de } \\
\text { correlación }\end{array}$ & 1.000 & $.474^{* *}$ \\
\hline & & Sig. (bilateral) & & 0.000 \\
\hline & & $\mathrm{N}$ & 250 & 250 \\
\hline & \multirow{3}{*}{ APDI } & $\begin{array}{c}\text { Coeficiente de } \\
\text { correlación }\end{array}$ & $.474^{* *}$ & 1.000 \\
\hline & & Sig. (bilateral) & 0.000 & \\
\hline & & $\mathrm{N}$ & 250 & 250 \\
\hline \multicolumn{5}{|c|}{ **. La correlación es significativa en el nivel 0,01 (bilateral). } \\
\hline
\end{tabular}

En la Tabla 8 se muestra el p-valor obtenido (0.000), con lo cual se determina la existencia de correlación entre las variables de estudio. También se analiza el coeficiente de Spearman, arrojando un resultado de 0.365 , considerado como una correlación positiva débil entre las variables analizadas.

Tabla 8. Correlación de Spearman entre el conocimiento transformación digital y la experiencia de uso herramientas transformación digital

Table 8. Spearman's correlation between digital transformation knowledge and the experience of using digital transformation tools

\begin{tabular}{|c|c|c|c|c|}
\hline & & & CONTD & EXTD \\
\hline \multirow{6}{*}{ Rho de Spearman } & & $\begin{array}{l}\text { Coeficiente de } \\
\text { correlación }\end{array}$ & 1.000 & $.365^{* *}$ \\
\hline & \multirow{2}{*}{ CONTD } & Sig. (bilateral) & & 0.000 \\
\hline & & $\mathrm{N}$ & 250 & 250 \\
\hline & \multirow{3}{*}{ EXTD } & $\begin{array}{c}\text { Coeficiente de } \\
\text { correlación }\end{array}$ & $.365^{* *}$ & 1.000 \\
\hline & & Sig. (bilateral) & 0.000 & \\
\hline & & N & 250 & 250 \\
\hline
\end{tabular}

**. La correlación es significativa en el nivel 0,01 (bilateral).

Fuente: elaboración propia.

En la Tabla 9 se muestra el p-valor obtenido (0.001), evidenciando la existencia de correlación entre las variables de estudio, con respecto al coeficiente de Spearman, el cual fue de 0.214 , se interpreta una correlación positiva débil entre las variables analizadas. 
Tabla 9. Correlación de Spearman entre el conocimiento transformación digital y la intención de uso en el futuro

Table 9. Spearman's correlation between digital transformation knowledge and the intention to use in the future

\begin{tabular}{|c|c|c|c|c|}
\hline & & & CONTD & INTUF \\
\hline \multirow{6}{*}{ Rho de Spearman } & \multirow{3}{*}{ CONTD } & $\begin{array}{c}\text { Coeficiente de } \\
\text { correlación }\end{array}$ & 1.000 & $.214^{* *}$ \\
\hline & & Sig. (bilateral) & & 0.001 \\
\hline & & $N$ & 250 & 250 \\
\hline & \multirow{3}{*}{ INTUF } & $\begin{array}{c}\text { Coeficiente de } \\
\text { correlación }\end{array}$ & $.214^{* *}$ & 1.000 \\
\hline & & Sig. (bilateral) & 0.001 & \\
\hline & & $N$ & 250 & 250 \\
\hline
\end{tabular}

\footnotetext{
**. La correlación es significativa en el nivel 0,01 (bilateral).

Fuente: elaboración propia.
}

En la Tabla 10 se muestra el p-valor obtenido (0.178), lo que evidencia la inexistencia de correlación entre las variables de estudio. También se analiza el coeficiente de Spearman, el cual fue de 0.085 , lo que significa que no existe correlación entre las variables de apropiación digital y la experiencia de uso de herramientas de transformación digital.

Tabla 10. Correlación de Spearman entre la apropiación digital y la experiencia de uso herramientas transformación digital

Table 10. Spearman's correlation between digital appropriation and the experience of using digital transformation tools

\begin{tabular}{|c|c|c|c|c|}
\hline & & & APDI & EXTD \\
\hline \multirow{6}{*}{ Rho de Spearman } & \multirow{3}{*}{ APDI } & $\begin{array}{ll}\begin{array}{l}\text { Coeficiente } \\
\text { correlación }\end{array} & \text { de } \\
\end{array}$ & 1.000 & 0.085 \\
\hline & & Sig. (bilateral) & & 0.178 \\
\hline & & $\mathrm{N}$ & 250 & 250 \\
\hline & \multirow{3}{*}{ EXTD } & $\begin{array}{l}\text { Coeficiente } \\
\text { correlación }\end{array}$ & 0.085 & 1.000 \\
\hline & & Sig. (bilateral) & 0.178 & \\
\hline & & $\mathrm{N}$ & 250 & 250 \\
\hline
\end{tabular}

**. La correlación es significativa en el nivel 0,01 (bilateral).

Fuente: elaboración propia.

En la Tabla 11 se muestra el p-valor obtenido (0.870), lo que evidencia la inexistencia de correlación entre las variables de estudio. También se analiza el coeficiente de Spearman, el cual fue de 0.010, lo que significa que no existe correlación entre las variables de apropiación digital y la intención de uso en el futuro. 
Tabla 11. Correlación de Spearman entre la apropiación digital y la intención de uso en el futuro Table 11. Spearman's correlation between digital appropriation and the intention to use in the future

\begin{tabular}{|c|c|c|c|c|}
\hline & & & APDI & INTUF \\
\hline \multirow{6}{*}{ Rho de Spearman } & \multirow{3}{*}{ APDI } & $\begin{array}{c}\text { Coeficiente de } \\
\text { correlación }\end{array}$ & 1.000 & 0.010 \\
\hline & & Sig. (bilateral) & & 0.870 \\
\hline & & $\mathrm{N}$ & 250 & 250 \\
\hline & \multirow{3}{*}{ INTUF } & $\begin{array}{c}\text { Coeficiente de } \\
\text { correlación }\end{array}$ & 0.010 & 1.000 \\
\hline & & Sig. (bilateral) & 0.870 & \\
\hline & & $\mathrm{N}$ & 250 & 250 \\
\hline \multicolumn{5}{|c|}{ **. La correlación es significativa en el nivel 0,01 (bilateral). } \\
\hline
\end{tabular}

La Tabla 12 evidencia el p-valor obtenido (0.000), valida la presencia de correlación entre las variables de estudio. De igual forma, se observa el coeficiente de Spearman, el cual fue de 0.597, lo que significa una correlación positiva moderada entre las variables analizadas.

Tabla 12. Correlación de Spearman entre la experiencia de uso herramientas transformación digital y la intención de uso en el futuro

Table 12. Spearman's correlation between the experience of using digital transformation tools and the intention to use in the future

\begin{tabular}{|c|c|c|c|c|}
\hline & & & EXTD & INTUF \\
\hline & \multirow{3}{*}{ EXTD } & $\begin{array}{l}\text { Coeficiente de } \\
\text { correlación }\end{array}$ & 1.000 & $.597^{* *}$ \\
\hline & & Sig. (bilateral) & & 0.000 \\
\hline \multirow{4}{*}{ Rho de Spearman } & & $\mathrm{N}$ & 250 & 250 \\
\hline & \multirow{3}{*}{ INTUF } & $\begin{array}{c}\text { Coeficiente de } \\
\text { correlación }\end{array}$ & $.597^{* *}$ & 1.000 \\
\hline & & Sig. (bilateral) & 0.000 & \\
\hline & & $N$ & 250 & 250 \\
\hline
\end{tabular}

**. La correlación es significativa en el nivel 0,01 (bilateral).

Fuente: elaboración propia.

La Tabla 13 resume los resultados del proceso de comprobación de hipótesis. 
Tabla 13. Resultado de hipótesis perfilamiento transformación digital

Table 13. Results of the hypothesis testing. Digital transformation profiling

\begin{tabular}{ccc}
\hline No $(\mathrm{H})$ & Hipótesis & Decisión \\
\hline $\mathrm{H} 1$ & $\begin{array}{c}\text { Existe una relación positiva entre el conocimiento sobre transformación } \\
\text { digital y apropiación de las nuevas tecnologías digitales. }\end{array}$ & Aceptada \\
\hline $\mathrm{H} 2$ & $\begin{array}{c}\text { Existe una relación positiva entre el conocimiento sobre transformación } \\
\text { digital y la experiencia de uso de herramientas digitales. }\end{array}$ & Aceptada \\
\hline $\mathrm{H} 3$ & $\begin{array}{c}\text { Existe una relación positiva entre el conocimiento sobre transformación } \\
\text { digital y la intención de uso en el futuro de herramientas digitales. }\end{array}$ & Aceptada \\
\hline $\mathrm{H} 4$ & $\begin{array}{c}\text { Existe una relación positiva entre la apropiación de las nuevas tecnologías } \\
\text { digitales y la experiencia de uso de herramientas digitales. }\end{array}$ & Rechazada \\
\hline $\mathrm{H} 5$ & $\begin{array}{c}\text { Existe una relación positiva entre la apropiación de las nuevas tecnologías } \\
\text { digitales y la intención de uso en el futuro de herramientas digitales. }\end{array}$ & Rechazada \\
\hline H6 & $\begin{array}{c}\text { Existe una relación positiva entre la experiencia en el uso de herramientas } \\
\text { digitales y la intención de uso en el futuro. }\end{array}$ & Aceptada
\end{tabular}

\section{DISCUSIÓN}

Esta investigación ayuda a comprender los factores que intervienen en la madurez tecnológica de la generación $Z$, definida aquí como alguien que proviene de un hogar y entornos enriquecidos por medios digitales, que, al igual que la investigación de Helsper y Eynon, 2010, se ha encontrado que la inmersión en un entorno digital (experiencia de uso) tiende a ser la variable más importante para determinar el nivel de madurez tecnológica, en algunos aspectos. Estos hallazgos también apoyan los argumentos presentados por Ifenthaler y Egloffstein, 2020, donde la interacción con herramientas digitales conlleva a una mayor apropiación de estas y en consecuencia incrementa el conocimiento sobre transformación digital.

Los hallazgos presentados en este estudio también apoyan los resultados encontrados por (Seong Leem et al., 2008), donde un modelo de madurez tecnológica dentro de una organización no solo depende de la infraestructura tecnológica ( $\mathrm{TI}$ ) o de las directivas de la empresa: el conocimiento y la experiencia de los participantes del proceso facilitará un mejor despliegue e implementación de la estrategia de transformación digital; de igual forma los hallazgos de esta investigación están alineados con los resultados de la investigación de Kaminskyi et al. 2018; Hernandez-de-Menendez et al. 2020, donde las universidades son, en gran parte, las responsables de ofrecer arquitecturas informáticas que mejoren el aprendizaje y desempeño tecnológico de la generación Z.

Por otra parte, la mayoría de estudios se centran en entender y desarrollar modelos de madurez tecnológica empresarial (Ifenthaler y Egloffstein, 2020; Kuzin, 2019; Seong Leem et al., 2008; Lutteroth et al., 2007; Maslennikova et al., 2019; Putri y Muljoredjo, 2014), donde se busca desarrollar unas fases que cualquier organización sin importar el sector puede consultar para descubrir cuál es su nivel de madurez tecnológica; por el contrario, esta investigación contribuye al área de administración y gestión tecnológica argumentando que el nivel de madurez tecnológica de la generación Z impacta de manera directa cualquier estrategia de transformación digital empresarial, donde uno de los hallazgos más relevantes fue determinar que la generación $Z$ requiere 
una experiencia de uso previa de tecnologías digitales para activar la intención de uso en el futuro de herramientas digitales. Este hallazgo es respaldado por la hipótesis sobre la relación que existe entre el conocimiento sobre la transformación digital y la intención de uso en el futuro.

Asimismo, un hallazgo importante que aporta esta investigación al sector empresarial fue identificar que no es suficiente tener un conocimiento teórico de las nuevas tecnologías digitales, los resultados demuestran que la experiencia de uso se relaciona fuertemente con la intención de uso en el futuro. Bajo este argumento se demuestra la necesidad de fortalecer el conocimiento practico en el uso de herramientas digitales, con lo cual se busca incrementar la intención de uso en el futuro y la participación en el diseño e implementación de estrategias de transformación digital en entornos laborales.

La investigación contribuye a las organizaciones que se encuentran en procesos de transformación digital, ya que aporta algunas variables que pueden ayudar a identificar el grado de madurez digital que tiene la generación $Z$, quienes serán los responsables en el futuro de utilizar las herramientas digitales que están inmersas en todos los procesos de la organización, pues la apropiación tecnológica no es suficiente para incentivar el uso de herramientas digitales, además de que la carencia de información relevante e importante y su aplicación en un entorno empresarial puede afectar la intención de uso por parte de la generación Z y, por supuesto, alterar el ecosistema digital de las organizaciones. Así las cosas, la apropiación digital debe estar concentrada en la aplicación de la tecnología en los diferentes procesos de la organización, lo que proporcionaría valor en la compresión y futuro uso de herramientas digitales.

Adicionalmente, uno de los aportes más importantes fue el de identificar la necesidad de fortalecer los procesos de aprendizaje a través de herramientas digitales aplicadas a entornos competitivos, lo que contribuirá al desempeño y desarrollo de competencias acordes a las necesidades empresariales de sectores que se encuentran en procesos de transformación digital a raíz de la COVID-19. La transformación digital ofrece oportunidades y desafíos tanto para los jóvenes de la generación Z, para empresas e instituciones de educación superior, que puedan contar con prospectos preparados para afrontar las nuevas tecnologías digitales y experimentar con ellas en entornos competitivos, lo que se constituye en algunos de los desafíos que se le presentan a las empresas y las IES; del mismo modo, la generación Z está comprendiendo el valor que proporcionan las tecnologías asociadas a la transformación digital, competitividad, eficiencia, eficacia, nivel de servicio, siendo estos algunos de los factores más importantes que aporta la transformación digital. En consideración, se debe incrementar el conocimiento y confianza en las nuevas tecnologías digitales, generando perdurabilidad y sostenibilidad de las organizaciones en Latinoamérica.

Por último, los resultados pueden haber sido impactos por el sesgo de autoselección. La muestra fue compuesta por personas ubicadas en la ciudad de Bogotá y con una alta interacción en entornos digitales, por lo tanto, los resultados deben interpretarse como una explicación de poblaciones con características similares; por otra parte, aunque la generación Z tiene patrones de comportamiento y poseen conocimientos tecnológicos similares, para poder generalizar los resultados en entornos económicos y sociales diferentes a los de la muestra se requerirá de investigación adicional. 


\section{CONCLUSIONES}

A partir de los resultados arrojados en esta investigación se puede concluir lo siguiente: primero, la generación Z está inclinada a la experiencia práctica de herramientas digitales, donde no es suficiente tener un conocimiento y comprensión teórica de la transformación digital; segundo, no existe una claridad de la aplicación práctica en los procesos empresariales de las herramientas vinculadas a un proceso de transformación digital, permitiendo disminuir la intención de formación y aprendizaje en nuevas tecnologías; tercero, dentro de los procesos de formación de los futuros responsables de administrar las nuevas tecnologías digitales se debe fortalecer el aprendizaje basado en retos, en función de nuevas tecnologías, por lo que no existe un interés de utilizar en el futuro tales herramientas para apoyar la toma de decisión.

La madurez digital de la generación Z se basa en el uso exclusivo de tecnologías de la comunicación, más no se centra en herramientas digitales de la industria 4.0, y estas son la base en procesos de transformación digital empresarial; en este orden de ideas, los responsables de formar esta generación solo se están preocupando por una apropiación conceptual y no práctica, lo que debilita el aprovechamiento de estas herramientas en un entorno competitivo.

Las organizaciones están realizando inversiones muy altas en procesos de transformación digital, lo cual hace necesario que establezcan un plan de adopción de herramientas digitales para las personas que van a administrar estos recursos; la curva de aprendizaje y experiencia está ocurriendo al interior de las organizaciones, desencadenando altos costos y pérdidas mayores, lo que resta competitividad, desperdiciando la oportunidad de aprovechar y maximizar las virtudes de las nuevas tecnologías digitales en un tiempo menor.

\section{CONFLICTOS DE INTERÉS}

Los autores declaran y confirman que no presentan conflictos de interés financiero, profesional o personal que pueda influir de forma inapropiada en los resultados obtenidos o las interpretaciones propuestas.

\section{CONTRIBUCIÓN DE AUTORES}

Para el desarrollo de este proyecto todos los autores han realizado una contribución significativa especificada a continuación:

Óscar Armando Mejía-Delgado: construcción de instrumento de investigación, recolección de datos, análisis estadístico de datos, discusión y conclusiones

Yuri Yasmin Mejía-Delgado: revisión de literatura, construcción de instrumento de investigación, recolección de datos, construcción del marco de referencia, discusión y conclusiones. 


\section{REFERENCIAS}

Adamik, A., Nowicki, M. (2018). Preparedness of companies for digital transformation and creating a competitive advantage in the age of Industry 4.0. Proceedings of the International Conference on Business Excellence, v. 12, n. 1, 10-24. https://doi.org/10.2478/picbe-2018-0003

Akçayir, M., Dündar, H., Akçayir, G. (2016). What makes you a digital native? Is it enough to be born after 1980? Computers in Human Behavior, v. 60, 435-440. https://doi.org/10.1016/j.chb.2016.02.089

Bharadwaj, A., El Sawy, O. A., Pavlou, P. A., Venkatraman, N. (2013). Digital business strategy: Toward a next generation of insights. MIS Quarterly, v. 37, n. 2, 471-482. URL

Blayone, T. J. B., Mykhailenko, O., Kavtaradze, M., Kokhan, M., vanOostveen, R., Barber, W. (2018). Profiling the digital readiness of higher education students for transformative online learning in the post-soviet nations of Georgia and Ukraine. International Journal of Educational Technology in Higher Education, v. 15, n. 37. https://doi.org/10.1186/s41239-018-0119-9

Bond, M., Marín, V. I., Dolch, C., Bedenlier, S., Zawacki-Richter, O. (2018). Digital transformation in German higher education: student and teacher perceptions and usage of digital media. International Journal of Educational Technology in Higher Education, v. 15, n. 48. https://doi.org/10.1186/s41239-018-0130-1

Churchill, G. A. (1979). A paradigm for developing better measures of marketing constructs. Journal of Marketing Research, v. 16, n. 1, 64-73. https://doi.org/10.2307/3150876

Conner, K. R., Prahalad, C. K. (1996). A Resource-based Theory of the Firm- Knowledge Versus Opportunism. Organization Science, v. 7, n. 5, 469-592. https://doi.org/10.1287/orsc.7.5.477

De Luca, L. M., Herhausen, D., Troilo, G., Rossi, A. (2021). How and when do big data investments pay off? The role of marketing affordances and service innovation. Journal of the Academy of Marketing Science, v. 49, n. 4, 790-810. https://doi.org/10.1007/s11747-020-00739-x

El Hilali, W., El Manouar, A., Janati Idrissi, M. A. (2020). Reaching sustainability during a digital transformation: a PLS approach. International Journal of Innovation Science, v. 12, n. 1, 52 79. https://doi.org/10.1108/IJIS-08-2019-0083

Fonseca, L. M. (2018). Industry 4.0 and the digital society: concepts, dimensions and envisioned benefits. Proceedings of the International Conference on Business Excellence, v. 12, n. 1, 386397. https://doi.org/10.2478/picbe-2018-0034

Gefen, D. (2000). E-commerce: The role of familiarity and trust. Omega, v. 28, n. 6, 725-737. https://doi.org/10.1016/S0305-0483(00)00021-9 
Gurung, B., Rutledge, D. (2014). Digital learners and the overlapping of their personal and educational digital engagement. Computers \& Education, v. 77, 91-100.

https://doi.org/10.1016/i.compedu.2014.04.012

Ha, S., Stoel, L. (2009). Consumer e-shopping acceptance: Antecedents in a technology acceptance model. Journal of Business Research, v. 62, n. 5, 565-571. https://doi.org/10.1016/i.jbusres.2008.06.016

Hadullo, K., Oboko, R., Omwenga, E. (2017). A model for evaluating e-learning systems quality in higher education in developing countries. International Journal of Education and Development using Information and Communication Technology, v. 13, n. 2, 185-204. URL

Hajkowicz, S., Reeson, A., Rudd, L., Bratanova, A., Hodgers, L., Mason, C., Boughen, N. (2016). Tomorrow's Digitally Enabled Workforce: Megatrends and scenarios for jobs and employment in Australia over the coming twenty years. Csiro. https://doi.org/10.4225/08/58557df808f71

Hausberg, J. P., Liere-Netheler, K., Packmohr, S., Pakura, S., Vogelsang, K. (2019). Research streams on digital transformation from a holistic business perspective: a systematic literature review and citation network analysis. Journal of Business Economics, n. 89, 931-963. https://doi.org/10.1007/s11573-019-00956-z

Helsper, E. J., Eynon, R. (2010). Digital natives: where is the evidence? British educational research journal, v. 36, n. 3, 503-520. https://doi.org/10.1080/01411920902989227

Hernandez-de-Menendez, M., Escobar Díaz, C. A., Morales-Menendez, R. (2020). Educational experiences with Generation Z. International Journal on Interactive Design and Manufacturing (IJIDeM), v. 14, n. 3, 847-859. https://doi.org/10.1007/s12008-020-00674-9

Hinings, B., Gegenhuber, T., Greenwood, R. (2018). Digital innovation and transformation: An institutional perspective. Information and Organization, v. 28, n. 1, 52-61. https://doi.org/10.1016/i.infoandorg.2018.02.004

Ifenthaler, D., Egloffstein, M. (2020). Development and Implementation of a Maturity Model of Digital Transformation, TechTrends, v. 64, 302-309. https://doi.org/10.1007/s11528-019-00457-4

Ipatov, O., Barinova, D., Odinokaya, M., Rubtsova, A., Pyatnitsky, A., (2020). The Impact of Digital Transformation Process of the Russian University. Proceedings of the 31st DAAAM International Symposium, pp. 0271-0275. https://doi.org/10.2507/31st.daaam.proceedings.037

Kaminskyi, O. Y., Yereshko, Y. O., Kyrychenko, S. O. (2018). Digital Transformation of University Education in Ukraine: Trajectories of Development in the Conditions of New Technological and Economic Order. Information Technologies and Learning Tools, v. 64, n. 2, 128- 137. https://doi.org/10.33407/itlt.v64i2.2083 
Kamsker, S., Janschitz, G., Monitzer, S. (2020). Digital Transformation and Higher Education: A Survey on the Digital Competencies of Learners to Develop Higher Education Teaching. International Journal for Business Education, n. 160, 22-41. URL

Kane, G. (2019). The Technology Fallacy: People Are the Real Key to Digital Transformation. Research Technology Management, v. 62, n. 6, 44-49. https://doi.org/10.1080/08956308.2019.1661079

Kisimov, V., Kabakchieva, D., Naydenov, A., Stefanova, K. (2020). Agile Elastic Desktop Corporate Architecture for Big Data. Cybernetics and Information Technologies, v. 20, n. 3, 15-31. https://doi.org/10.2478/cait-2020-0025

Kumar, N., Kumar, G., Singh, R. K. (2021). Big data analytics application for sustainable manufacturing operations: analysis of strategic factors. Clean Technologies and Environmental Policy, v. 23, 965-989. https://doi.org/10.1007/s10098-020-02008-5

Kuzin, D. V. (2019). Problems of Digital Maturity in Modern Business. The World of New Economy, v.13, n. 3, 89-99. https://doi.org/10.26794/2220-6469-2019-13-3-89-99

LeBlanc, P. J. (2018). Higher Education in a VUCA World. Change: The Magazine of Higher Learning, v. 50, n. 3-4, 23-26. https://doi.org/10.1080/00091383.2018.1507370

Lutteroth, C., Luxton-Reilly, A., Dobbie, G., Hamer, J. (2007). A maturity model for computing education. Conferences in Research and Practice in Information Technology Series, 66, 107114.

Machika, P. C., Dolley, F. (2018). Framework for a learning management system at a university of technology with a weak information technology maturity system. South African Journal of Higher Education, v. 32, n. 2, 176-191. https://doi.org/10.20853/32-2-1502

Maslennikova, S. V., Zhurakovskaya, V. M., Bogovarova, V. A., Moiseev, R. (2019). Cognitive Modelling of the Socialization Process as a Digital Transformation Trend in Higher Education. $V$ International Forum on Teacher Education, 1105-1112. https://doi.org/10.3897/ap.1.e1049

Matt, C. (2020). Some Remarks on the Challenges of Digital Transformation Research in the Business Sectors. Trilogía Ciencia Tecnología Sociedad, v. 12, n. 23, 7-10. https://doi.org/10.22430/21457778.1708

Matt, C., Hess, T., Benlian, A. (2015). Digital Transformation Strategies. Business and Information Systems Engineering, v. 57, n. 5, 339-343. https://doi.org/10.1007/s12599-015-0401-5

Matt, C., Hess, T., Benlian, A., Wiesbock, F., (2016). Options for Formulating a Digital Transformation Strategy. MIS Quarterly Executive, v. 15, n. 2. URL 
Mugge, P., Abbu, H., Michaelis, T. L., Kwiatkowski, A., Gudergan, G. (2020). Patterns of digitization: A practical guide to digital transformation. Research-Technology Management, v. 63, n. 2, 2735. https://doi.org/10.1080/08956308.2020.1707003

Newman, T., Beetham, H. (2017). Student digital experience tracker 2017: The voice of 22,000 UK learners. Bristol.

Pappas, I. O., Mikalef, P., Giannakos, M. N., Krogstie, J., Lekakos, G. (2018). Big data and business analytics ecosystems: paving the way towards digital transformation and sustainable societies. Information Systems and E-Business Management, v. 16, 479-491. URL

Putri, N. K. S., Muljoredjo, H. (2014). The Use Of Green Information Technology Governance Model To Determine Capability Maturity Level In Dki Jakarta Private Higher Education Institutions. Journal of Theoretical and Applied Information Technology, v. 61, n. 1, 1-9. URL

Redecker, C. (2017). European Framework for the Digital Competence of Educators: DigCompEdu. JRC Publications Repository. URL

Roumani, Y., Nwankpa, J. K., Roumani, Y. F. (2015). The impact of incentives on the intention to try a new technology. Technology Analysis and Strategic Management, v. 27, n. 2, 126-141. https://doi.org/10.1080/09537325.2014.952625

Seong Leem, C., Kim, I. (2004). An integrated evaluation system based on the continuous improvement model of IS performance. Industrial Management \& Data Systems, v. 104, n. 2. 115-128. https://doi.org/10.1108/02635570410522080

Seong Leem, C., Wan Kim, B., Jung Yu, E., Ho Paek, M. (2008). Information technology maturity stages and enterprise benchmarking: an empirical study. Industrial Management \& Data Systems, v. 108, n. 9, 1200-1218. https://doi.org/10.1108/02635570810914892

Stjepić, A.-M., Ivančić, L., Vugec, D. S. (2020). Mastering digital transformation through business process management: Investigating alignments, goals, orchestration, and roles. Journal of Entrepreneurship, Management and Innovation, v. 16, n. 1, 41-74. https://doi.org/10.7341/20201612

Verčič, A. T., Verčič, D. (2013). Digital natives and social media. Public Relations Review, v. 39, n. 5, 600-602. https://doi.org/10.1016/i.pubrev.2013.08.008

Zhou, G., Xu, J. (2007). Adoption of Educational Technology: How Does Gender Matter? International Journal of Teaching and Learning in Higher Education, v. 19, n. 2, 140-153. URL 